\title{
Robotically enhanced coronary artery bypass surgery
}

\author{
Yugal K. Mishra $\cdot$ H. Wasir · Malhotra Rajneesh $\cdot$ \\ K. K. Sharma $\cdot$ Y. Mehta $\cdot$ N. Trehan
}

Received: 4 January 2007 / Accepted: 4 June 2007 / Published online: 13 July 2007

(C) Springer London 2007

\begin{abstract}
Robotically enhanced telemanipulation surgery is a rapidly developing technique which enables totally endoscopic cardiac surgery with utmost precision and perfection on both beating heart and arrested heart. Between December 2002 and September 2006, 268 patients underwent robotically enhanced coronary artery bypass surgery using the da Vinci telemanipulation system. Fourteen patients underwent total endoscopic coronary artery bypass surgery. Of these 12 were performed on a beating heart and 2 on an arrested heart. Two-hundred and fiftyfour patients had endoscopic takedown of the internal mammary artery followed by minimally invasive direct coronary artery bypass in 193 patients and left anterolateral thoracotomy in 61 patients. The internal mammary artery mobilization time was $36 \mathrm{~min}(28-76 \mathrm{~min})$ and the left internal mammary artery to left anterior descending artery anastomosis time ranged from 20 to $36 \mathrm{~min}$ for the totally endoscopic coronary artery bypass patients. The right internal mammary artery of one patient was anastomosed to diagonal artery totally endoscopically. The mean internal mammary artery flow by Doppler measurement in patients undergoing minimally invasive direct coronary artery bypass was $58 \mathrm{ml} \mathrm{min}{ }^{-1}$. Seven patients required conversion to median sternotomy and coronary bypass
\end{abstract}

Y. K. Mishra $(\bowtie) \cdot$ H. Wasir · M. Rajneesh $\cdot$ N. Trehan

Department of Cardiovascular Surgery,

Escorts Heart Institute and Research Centre,

Okhla Road, New Delhi 110025, India

e-mail: dryugal@yahoo.com

K. K. Sharma · Y. Mehta

Department of Cardiovascular Anesthesiology,

Escorts Heart Institute and Research Centre, New Delhi, India surgery on the beating heart. The mean intensive care unit stay was 1.2 days and the mean hospital stay 4.5 days. There was one in-hospital mortality. All 14 patients who underwent total endoscopic bypass surgery had coronary angiography 3 months later which showed $100 \%$ patency in 13 patients. One patient had 50\% anastomotic narrowing for which coronary angioplasty was performed in the same sitting. By using telematic technology, a complete endoscopic anastomosis is possible in both single vessels and suitable double vessel disease patients. The use of robotics is now extended to achieve complete myocardial revascularization by harvesting both the internal mammary arteries and making a small thoracotomy for direct anastomosis also.

Keywords Coronary artery bypass grafting · Coronary artery disease $\cdot$ Minimally invasive surgery

\section{Introduction}

The ultimate goal of minimally invasive coronary artery bypass grafting $(\mathrm{CABG})$ is to perform the entire anastomosis endoscopically. This is done by keeping two main concepts of minimally invasive surgery in mind: first, to circumvent the deleterious effect of cardiopulmonary bypass (CPB); and, second, to minimize the incision and surgical trauma. The objectives are also to reduce patient morbidity, length of hospital stay, and overall cost. Minimally invasive direct coronary artery bypass (MID$\mathrm{CAB}$ ) via a small ministernotomy approach or a 2 -in. left anterior thoracotomy approach, also termed endoscopic atraumatic coronary artery bypass (endoACAB) [1, 2], refers to such approaches. Advances in beating heart surgery has also aided these approaches. In patients for multivessel 
revascularization where direct aortovenous cannulation was not possible through a limited incision, the port-access system via femoral approach (Heartport, Redwood City, CA, USA) is very helpful for establishing CPB [3-5].

With the advent of robotically enhanced telemanipulation, the latest in minimally invasive techniques is now available and thus enables true closed-chest totally endoscopic procedures [6-9]. Our institute initiated the robotic program using the da Vinci surgical system (Intuitive Surgical, Mountain View, CA, USA) in December 2002. We review our experiences with the use of da Vinci in performing $\mathrm{CABG}$ using various minimally invasive approaches.

\section{Methods}

Between December 2002 and September 2006, 268 patients underwent robotically enhanced CABG. Fourteen patients underwent totally endoscopic coronary artery bypass (TECAB). Of these, 12 were done on the beating heart and 2 on the arrested heart using the Port Access System. Thirteen patients who underwent TECAB had single-vessel left anterior descending (LAD) disease and one patient had single-vessel disease with additional proximal diagonal artery disease for which anastomosis was done using the right internal mammary artery on the beating heart.

Two-hundred and fifty-four underwent endoACAB in which the skeletonized internal mammary was harvested in its entire length robotically and direct anastomosis was performed either by MIDCAB $(n=193)$ or using a small anterior thoracotomy incision (THORACAB) $(n=61)$. All patients were informed about the procedure preoperatively and each patient gave a written informed consent; institutional review board permission was also obtained. The 268 patients (213 males and 55 females) were $56.2 \pm 6.4$ years old (34-78 years). The preoperative demographic profile is listed in Table 1. Left ventricular ejection fraction (LVEF) was $48 \pm 1.6 \%$ and New York Heart Association (NYHA) class was $2.4 \pm 0.4$. The prerequisites for inclusion of patients for any robotic procedure were that they required CABG, were 18-80 years of age, and gave informed consent to the procedure.

Patients with significant co-morbidity including compromised pulmonary function tests, those requiring additional cardiac procedures, LVEF $<30 \%$, associated ventricular or aortic aneurysm surgery or those with significant peripheral vascular disease, thus making them unfit for port access procedures, were not considered for robotic surgery. Other preoperative exclusion criteria included body mass index $>35.0 \mathrm{~kg} \mathrm{~m}^{-2}$, decompensated congestive heart failure (NYHA III, IV), acute pulmonary edema, uncontrolled hypertension, any coagulopathy or history of acute myocardial infarction of less than 30 days' duration.
Table 1 Preoperative demographic profile $(n=268)$

\begin{tabular}{ll}
\hline Age & $56.2 \pm 6.4(34-78$ years $)$ \\
Male & 213 \\
Female & 55 \\
NIDDM & $80(30 \%)$ \\
Hypertension & $107(46.0 \%)$ \\
Smoking & $67(25 \%)$ \\
COPD & $19(7 \%)$ \\
LVEF & $48 \pm 1.6 \%$ \\
Stable angina & $134(50 \%)$ \\
Previous MI & $32(12 \%)$ \\
NYHA class & $2.4 \pm 0.4$ \\
\hline
\end{tabular}

NIDDM, non-insulin-dependent diabetes mellitus; COPD, chronic obstructive pulmonary disease; LVEF, left ventricular ejection fraction; MI, myocardial infarction; NYHA, New York Heart Association

Intraoperatively, the procedure was converted to conventional $\mathrm{CABG}$ on beating heart if any of the following criteria were found: systolic arterial pressure drop of $>20 \%$ of baseline or $<80 \mathrm{mmHg}$ for $>15 \mathrm{~min}$ or not responding to therapy; dense pleural or pericardial adhesions limiting visibility; intramyocardial LAD or target LAD diameter $<1.5 \mathrm{~mm}$ previously not identified on coronary angiogram; or inadequate visualization or malpresentation of target vessel such that it was not accessible via routine endoscopic ports.

\section{Anesthesia for TECAB}

After routine induction of anesthesia the patient underwent double-lumen intubation for single right lung ventilation. Arterial cannulation was performed using both radial and femoral arteries. Bilateral radial cannulation was performed for invasive monitoring of the endoaortic occlusion catheter in patients who underwent TECAB on an arrested heart. All patients had thermodilution pulmonary artery catheter monitoring and transesophageal echocardiography (TEE) for assessment of cardiac function and for positioning and monitoring the endoaortic catheter of the port-access system.

The da Vinci surgical system

The da Vinci telemanipulation system consists of a master console (Fig. 1) used by the operating surgeon for controlling the micro instruments which are mounted on the surgical cart, which has three arms (slave unit, Fig. 2). The surgical assistant manages changing of the instruments and positioning of the arms and camera on the slave unit. Of the three arms the left and right arms carrying the microinstruments resemble the human wrist and correspond to the left 


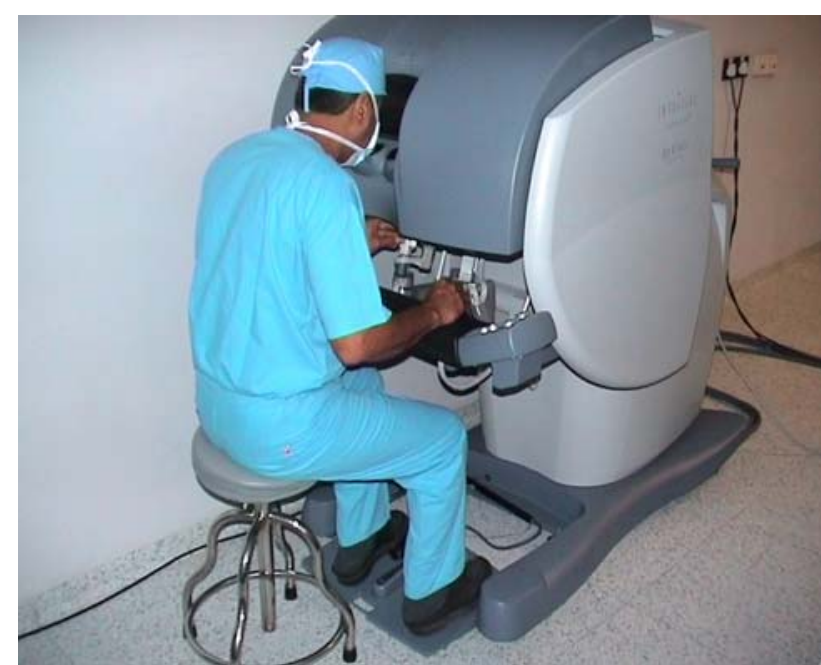

Fig. 1 Robotic master console

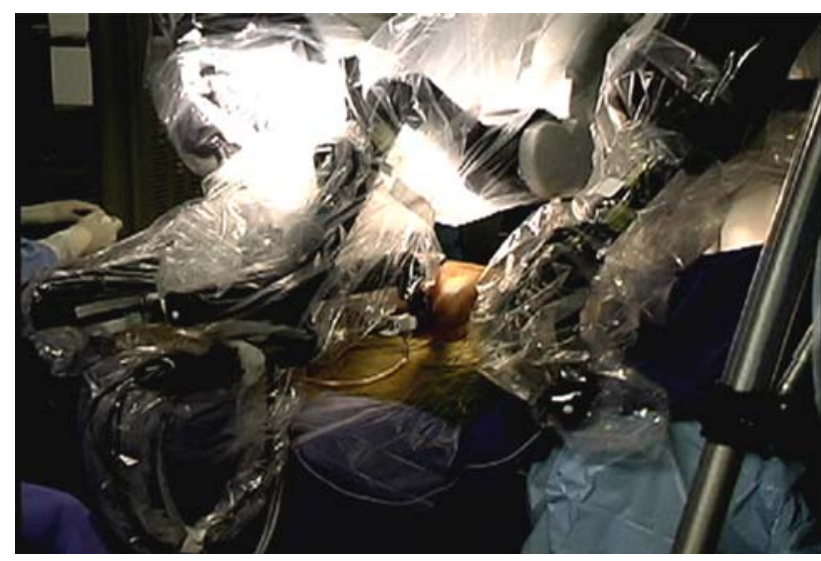

Fig. 2 Robotic cart or slave unit

and right arms on the main console. The middle arm carries a stereo endoscope for three-dimensional vision seen at the console end. An additional video cart carries the light source, a continuous carbon dioxide insufflator, and a conventional two-dimensional monitor for viewing by the surgical team.

The video image from the camera is transferred to the master console, magnified $(\times 10)$, and projected as a $3 \mathrm{D}$ image for optimum visualization.

\section{Operative technique for TECAB}

The approach for both the left internal mammary artery (LIMA) and the right internal mammary artery (RIMA) can be from the left pleural cavity. The patient is placed in the supine position with the left chest elevated by approximately $30^{\circ}$. Landmarks such as suprasternal notch, xiphoid, and second to sixth ribs are marked. After ensuring single right lung ventilation the camera cannula with $\mathrm{CO}_{2}$ insufflator port is inserted in the fourth or fifth intercostal space close to the anterior axillary line and the chest is insufflated with warm $\mathrm{CO}_{2}$ at $37^{\circ} \mathrm{C}$. After insertion of the endoscope, two ports are placed under visual control to accommodate the two robot arms, usually in the second and sixth intercostal spaces. LIMA is harvested skeletonized right from its origin from the left subclavian artery to its bifurcation using a $30^{\circ}$ angled endoscope facing upwards (Fig. 3). Unipolar cautery is used at low intensity for the LIMA branches. RIMA can be approached via the same ports after creating a retrosternal mediastinal plane and opening the right pleura. The pericardium is then opened using the same endoscopies with a $30^{\circ}$ down position for visualization of the LAD and its target site for anastomosis. If TECAB is proposed on an arrested heart the port-access system is used to initiate $\mathrm{CPB}$ and infusion of antegrade cardioplegia into the aortic root via the port-access endoclamp enables reliable cardiac arrest.

For the beating heart, when the internal mammary artery (IMA) is harvested the endostabilizer is inserted via a fourth port in the xiphoid area under endoscopic vision. The patient is thereafter heparinized and the IMA prepared for anastomosis. Throughout the procedure the $\mathrm{CO}_{2}$ pressure is kept between 10 and $14 \mathrm{mmHg}$ depending on the working space, at the same time monitoring the systolic pressure for any hemodynamic instability.

When the LAD is opened the anastomosis is performed either by using continuous endoscopic suture or by using endoclips (US Surgical, Connecticut, USA) (Fig. 4).

After completion of anastomosis the endoaortic occlusion catheter is deflated and the patient weaned from CPB. Heparin is reversed using protamine and a single chest tube is placed via the xiphoid port which is guided into the left pleural cavity.

In patients for whom MIDCAB or endo $A C A B$ was performed to LAD the approach was either via a small ministernotomy or a 2.5-in. left anterior thoracotomy incision after a robotic takedown of skeletonized LIMA. For all

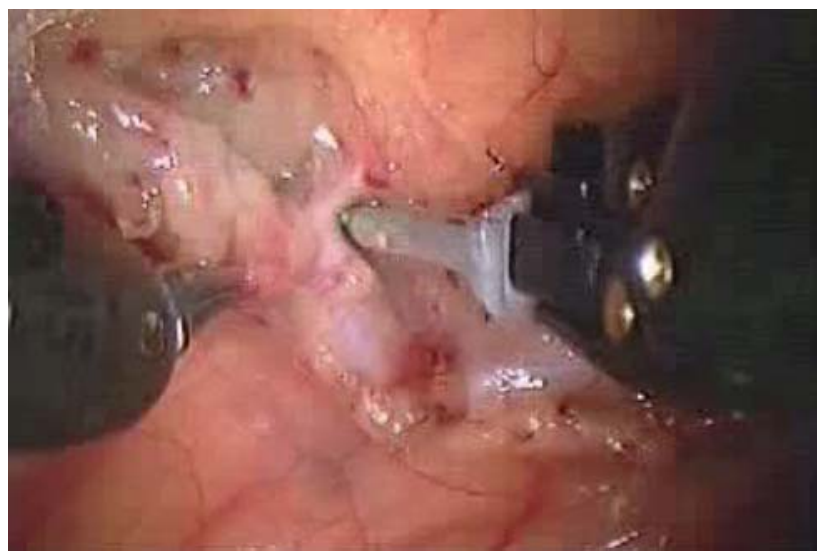

Fig. 3 Skeletonized left internal mammary artery dissection 


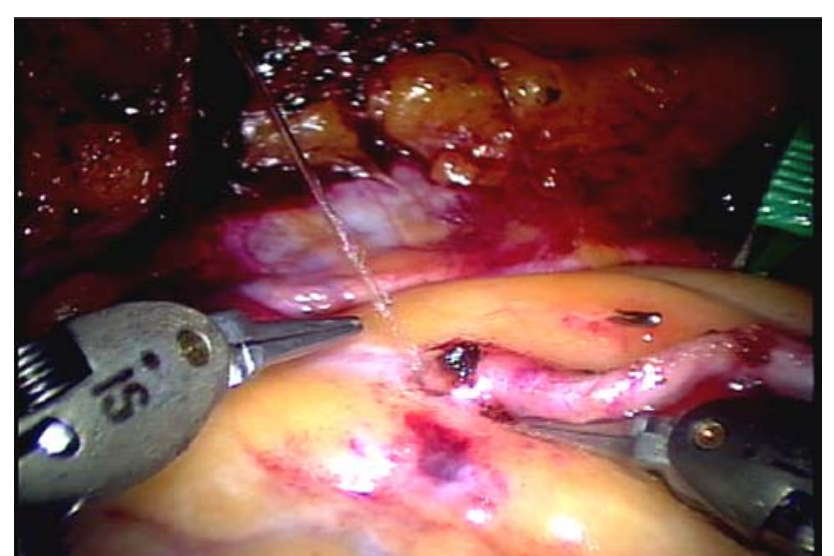

Fig. 4 Robotic LIMA to LAD anastomosis using endoclips

patients the LIMA flow was checked by the Doppler flow method when the anastomosis was complete and before protamine reversal.

\section{Results}

Two-hundred and sixty-eight patients underwent robotic enhanced CABG. Twelve TECAB were performed on beating hearts of which 11 were for single-vessel LAD disease and 1 was for single-vessel disease in which RIMA was anastomosed to diagonal and LIMA to LAD on the beating heart. In 254 patients the LIMA or RIMA was harvested robotically in a skeletonized fashion. Of these, 61 underwent THORACAB via a small left anterior thoractomy incision. For TECAB patients the total operating time was $168 \pm 26 \mathrm{~min}$, in which the LIMA was mobilized in $36 \mathrm{~min}$ (range 28-76 min) and the LIMA to LAD anastomosis ranged from 20 to $36 \mathrm{~min}$. The LIMA mobilization time range was the same for the other patients. Table 2 shows the different intraoperative data. Seven patients had to be converted to conventional CABG. None of these were from the TECAB group. Of these, five patients had dense pleural adhesions and two patients developed hemodynamic instability not responding to medical treatment. In both patients there was a fall in systolic blood pressure with a rise in pulmonary artery pressure and a fall in oxygen saturation which was because of high $\mathrm{CO}_{2}$ insufflation pressures. There was no electrocardiographic ST-T (EKG) change or arrhythmias and once the patient was put back to two-lung ventilation all hemodynamic measurements became stable. All six had regular sternotomy and CABG was performed on the beating heart. No conversion for any hemodynamic instability has, however, been performed in the last 164 cases.

The postoperative data are summarized in Table 3. Three patients developed superficial infection of the anterior tho-
Table 2 Intra-operative data

\begin{tabular}{lll}
\hline & $\begin{array}{l}\text { Duration } \\
(\mathrm{min})\end{array}$ & $\begin{array}{l}\text { Range } \\
(\mathrm{min})\end{array}$ \\
\hline OR time & $168 \pm 26$ & $145-296$ \\
CPB time & 64 & - \\
Cross clamp time & 44 & - \\
IMA mobilization time (TECAB) & 36 & $28-76$ \\
LAD identification/dissection & 1.7 & $1-3$ \\
$\begin{array}{l}\text { LIMA-LAD anastomosis time } \\
\quad \text { TECAB) }\end{array}$ & & $20-36$ \\
$\begin{array}{l}\text { RIMA-diag anastomosis time } \\
\quad(\text { TECAB) }\end{array}$ & 32 & - \\
$\begin{array}{l}\text { IMA flow (MIDCAB) } \\
\text { Pulsatility index PI (MIDCAB) }\end{array}$ & 2.7 & $(1.4-3.6)(N<5)$ \\
Conversion to median sternotomy & 7 & - \\
$\quad$ and OPCAB (MIDCAB) & & \\
\hline
\end{tabular}

OR, operating room; CPB, cardiopulmonary bypass; IMA, internal mammary artery; LAD, left anterior descending artery; LIMA, left internal mammary artery; RIMA, right internal mammary artery; OP$\mathrm{CAB}$, off pump coronary artery bypass

racotomy site which responded to antibiotics. These patients who had undergone MIDCAB were re-explored for bleeding. Re-exploration was done via the same limited incision. In two patients the bleeding was from the mammary branch; in the third patient, however, no specific bleeding site was found. There was one mortality in the non-TECAB group. This patient, who had undergone MID$\mathrm{CAB}$, was a known case of cholelithiasis who developed fulminant gall stone pancreatitis postoperatively.

Follow-up data are summarized in Table 4. All the patients who underwent TECAB had an angiogram on follow up at 3 months. Of these 14 TECAB patients there was $100 \%$ anastomotic patency (Fig. 5) in 13 patients and 1 patient had 50\% anastomotic narrowing for which angioplasty was performed at the same sitting.

Table 3 Postoperative data

\begin{tabular}{ll}
\hline Total ventilation time $(\mathrm{h})$ & 4.24 \\
Chest tube drainage & $140(55 \mathrm{ml}-360 \mathrm{ml})$ \\
ICU stay & 1.2 days \\
Hospital stay & 4.5 days \\
Perioperative MI (CPK-MB > 10\% & Nil \\
$\quad$ of CPK) 6 h postoperative sample & \\
Re-operation for bleeding & 3 \\
New onset atrial fibrillation & Nil \\
Wound infection & 1 \\
Mortality & 1 \\
\hline
\end{tabular}

ICU, intensive care unit; MI, myocardial infarction 
Table 4 Follow-up data

\begin{tabular}{ll}
\hline Duration & $1-42$ months \\
Late mortality & Nil \\
Recurrence of angina & Nil \\
Patient follow-up & \\
TECAB & $100 \%$ \\
Rest & $86.8 \%$ \\
Postoperative angiogram (3 months) & 14 \\
LIMA-LAD patency & 13 \\
\hline
\end{tabular}

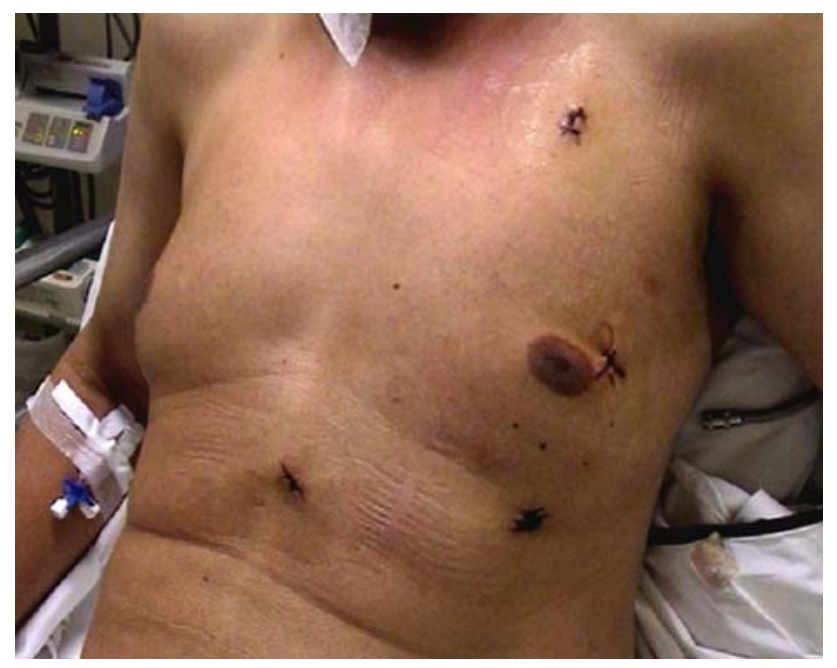

Fig. 5 Postoperative scars in TECAB patient

\section{Discussion}

The treatment of single-vessel coronary artery disease is usually non-surgical. When surgery is indicated in patients with double-vessel disease and a suitable anatomy, however, one always wants the least invasive procedure with the objective of a smaller incision to minimize surgical trauma, improve cosmesis, reduce risk of infection, and cause the least postoperative pain. With MIDCAB and OPCAB being established techniques, morbidity was reduced from conventional $\mathrm{CABG}$ on $\mathrm{CPB}$. With development of femoral port-access technology for peripheral CPB induction, the skin incision could be reduced further [10, 11] (Fig. 5). TECAB using robotic telemanipulators finally revolutionized the minimally invasive techniques of CABG $[6,8]$. If performed on the arrested heart the port-access technique is mandatory. Our experience with port-access surgery, which we have been routinely performing for ASD closure and mitral valve surgery, was helpful, as was our experience on beating heart surgery which we now apply routinely for most CABGs. Our CPB time of $64 \mathrm{~min}$ and crossclamp time of $44 \mathrm{~min}$ is comparable with those of other centers performing TECAB mostly on arrested hearts [6].

We believe there should be a low threshold for conversion to the open technique in the initial stages of robotic surgery to reduce patient risk. Although pleural and pericardial adhesiolysis is very much possible, unless sufficient experience is gained with this technique it should be avoided.

Although adequate port placement was possible for all patients, because each patient has a different chest anatomy proper marking of port sites is of utmost important to avoid unnecessary struggle because of instrument collision and inability to perform complete IMA takedown because of left shoulder or subcostal interference. This is more so for patients who have medium-sized chest cavities, for extremely obese males, or for females with large breasts. We believe these patients will benefit from an endoscopic procedure, because the risk of wound infection is almost nil, as seen in our series. The feasibility and safety of closed-chest IMA takedown has been shown by several other groups $[7,12,13]$. Although use of TECAB can be extended to patients with double-vessel disease, as performed for one of our patients where RIMA was anastomosed to a large diagonal, suitable anatomy is essential before going ahead. Dogan et al. [6] reviewed their experience with double-vessel disease using both isolated IMAs and sequential anastomosis [9].

Bilateral IMA grafting is feasible but seems to be challenging and time-consuming, and therefore has a very limited indication. The use of multidetector CT scanning in determining the exact position of coronary target site and size for anastomosis using TECAB has also been practiced [6].

Although all our patients were ambulated by the first postoperative day and most were fit for discharge by the second postoperative day, as part of a protocol they were discharged by the fourth postoperative day and came for first follow-up on the seventh postoperative day.

The ultimate success of the robotic procedure is a combined effort of the surgeon at the console, the assistant surgeon at the slave cart, the anesthesiologist and the perfusionist, all of whom need to work as a team with communication at every stage. From the change of instruments to hemodynamic monitoring, the $\mathrm{CO}_{2}$ pressures and routine blood gas monitoring all require utmost vigilance at all times to perform this minimally invasive procedures to perfection.

The hemodynamics of single lung ventilation and anesthesiologic management of all patients undergoing robotic surgery are increasingly important in cardiac anesthesia [14]. Hypotension, secondary to raised intrathoracic pressure due to $\mathrm{CO}_{2}$ insufflation must be controlled meticulously.

Although most centers continue to perform TECAB on arrested hearts it is still premature to comment on the quality 
of anastomosis on beating versus arrested heart. With our experience of beating heart surgery-our results from beating heart TECAB — we believe that TECAB performed on a beating heart is a safe option which circumvents the unsafe effects of CPB, especially if used as a new procedure. As with all other emerging technology, the learning curve is gradual and a word of caution is essential for all beginners.

In time, the present limitations of the system, for example an additional degree of freedom inside the chest cavity, additional support of the beating heart, and approachability to different target vessels, will be overcome. The addition of the third arm and the next version of the robot with vibrotactile sensation while grasping and suturing will be a step forward in the developing technology.

\section{Conclusions}

The use of telematic technology is feasible for performing CABG on arrested and beating hearts, with good postoperative results. This can also be extended to achieve complete myocardial revascularization by bilateral robotic IMA takedown and direct anastomosis via small thoracotomy.

\section{References}

1. Talwalkar NG, Cooley DA (1998) Minimally invasive cardiac artery bypass grafting: a review. Cardiol Rev 6:345-349

2. Diegeler A (1999) Left internal mammary artery grafting to left anterior descending coronary artery by minimally invasive direct coronary artery bypass approach. Curr Cardiol Rep 1:323-330

3. Groh M, Grossi EA (1999) Multivessel coronary bypass grafting with minimal access using cardiopulmonary bypass. Curr Cardiol Rep 1:331-334
4. Wimmer-Greinecker G, Matheis G, Dogan S, Aybek T, Mierdl S, Kessler P et al (1999) Patient selection for Port-Access multi vessel revascularization. Eur J Cardiothorac Surg 16(Suppl 2):S43S47

5. Wimmer-Greinecker G, Matheis G, Dogan S, Aybek T, Kessler P, Westphal K et al (1999) Complications of port-access cardiac surgery. J Card Surg 14:240-245

6. Dogan S, Aybek T, Andressen E, Byhahn C, Mierdl S, Westphal $\mathrm{K}$ et al (2002) Totally endoscopic coronary artery bypass grafting on cardiopulmonary bypass with robotically enhanced telemanipulation: report of forty five cases. J Thorac Cardiovasc Surg 123:1125-1131

7. Loulmet D, Carpentier A, d'Attellis N, Berrebi A, Cardon C, Ponzio O et al (1999) Endoscopic coronary artery bypass grafting with the aid of robotic assisted instruments. J Thorac Cardiovasc Surg 118:4-10

8. Falk V, Diegeler A, Walther T, Banush J, Brucerius J, Raumans J et al (2000) Total endoscopic computer enhanced coronary artery bypass grafting. Eur J Cardiothorac Surg 17:38-45

9. Dogan S, Aybek T, Westphal K, Mierdl S, Moritiz A, WimmerGreinecker G (2001) Computer-enhanced totally endoscopic sequential arterial coronary artery bypass. Ann Thorac Surg 72:610-611

10. Groh MA, Sutherland SE, Burton HG, Johnson AM, Ely SW (1999) Port access coronary artery bypass grafting: technique and comparative results. Ann Thorac Surg 68:1506-1508

11. Galloway AC, Shemin RJ, Glower DD, Boyer JH Jr, Groh MA, Kuntz RE et al (1999) First report of port access international registry. Ann Thorac Surg 67:51-58

12. Falk V, Diegeler A, Walther T, Loscher N, Vogel B, Ulmann C et al (1999) Endoscopic coronary artery bypass grafting on the beating heart using a computer enhanced telemanipulation system. Heart Surg Forum 2(3):199-205

13. Falk V, Gummert JF, Walther T, Hayase M, Berry GJ, Mohr FW (1999) Quality of computer enhanced totally endoscopic coronary bypass graft anastomosis-comparison to conventional technique. Eur J Cardio Thorac Surg 15:260-265

14. Mehta Y, Gupta A (2003) Robotic surgery and anesthesia. The Indian Anesthes Forum, vol 2 (on-line journal) 